\title{
Julias Balkon
}

Manfred Pfister (FU Berlin)

I.

„Julias Balkon“ - was für ein kleines Thema für eine so großformatige Ringvorlesung! Und doch erweist es sich, schaut man nur genauer hin, als Welten und Zeiten überspannend. Lassen Sie mich weit ab von Europa beginnen, auf einer Spanisch sprechenden Insel der Karibik, auf Kuba. Und lassen Sie mich nicht mit einem Werk hoher Kunst beginnen, sondern mit einem materiellen Objekt der Alltagskultur: einem Zigarrenkistchen, das die stolze Aufschrift „Romeo y Julieta“ trägt und mit einem Bild von Julias Balkon und Romeos kühnen Aufschwung auf dessen Höhen verziert ist. Mein Kistchen ist aus den 70er Jahren, und sein Design stammt aus den zwanziger Jahren und ist seitdem im Wesentlichen unverändert geblieben ${ }^{1}$.

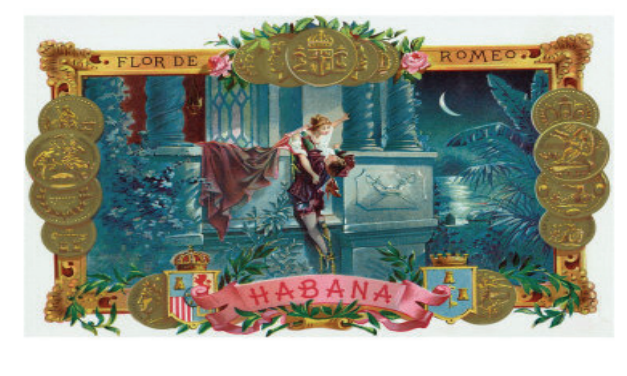

\footnotetext{
${ }^{1}$ Foto aus eigener Kollektion.
} 
Wie, fragt man sich, und warum sind die beiden romantischen Liebenden hier hingeraten? Nun, sie legen zumindest schon Zeugnis ab für den globalen Ruhm Shakespeares und seines italienischen Liebespaars bereits in den 1870er Jahren, als diese Sorte erlesener Kreation von Havannas sich mit ihrem Namen zu schmücken begann. Das war also nicht erst, als dem enthusiastischsten ihrer britischen Raucher, dem Shakespeare-Verehrer und Kriegsheld Winston Churchill, anlässlich seines Besuchs in La Habana 1946 die Ehre zu Teil wurde, seinem Lieblingsformat dieser Zigarre, Romeo y Julieta No 2, 18 Zentimeter lang und 18 Millimeter dick, den eigenen Namen verleihen zu dürfen, und so fürderhin seinen Namen auf der Bauchbinde mit dem des romantischen Paars zu vereinigen. Jeder freudianische Erklärungsversuch, der die phallische Form der Zigarre mit der legendären Potenz des britischen Premiers - oder darüber hinaus gar mit dem sexuellen Drive der jungen Liebenden - verbinden will, scheitert also schon an der Chronologie: Der Name war schon 70 Jahre vor ihm da. Wir müssen daher etwas tiefer in die Sozialgeschichte der kubanischen Zigarrenmanufaktur hinabsteigen, um eine plausiblere Antwort auf unsere Frage zu finden. ${ }^{2}$

Schon in der zweiten Hälfte des 19. Jahrhunderts galten die Arbeiter der kubanischen Zigarrenfabriken als die intellektuelle Elite unter dem städtischen Proletariat, und als solche leisteten sie sich auf eigene Kosten Vorleser, von denen sie sich während ihrer langen und eintönigen Arbeit durch das Vorlesen von Texten geistig anregen ließen. Es ging ihnen dabei also nicht in erster Linie um Unterhaltung, sondern um intellektuelle Aufrüstung für den Kampf gegen die spanischen Kolonisatoren in einer „tribuna avanzada de la libertad“, wie das der kubanische Revolutionsdichter José Martí nannte.

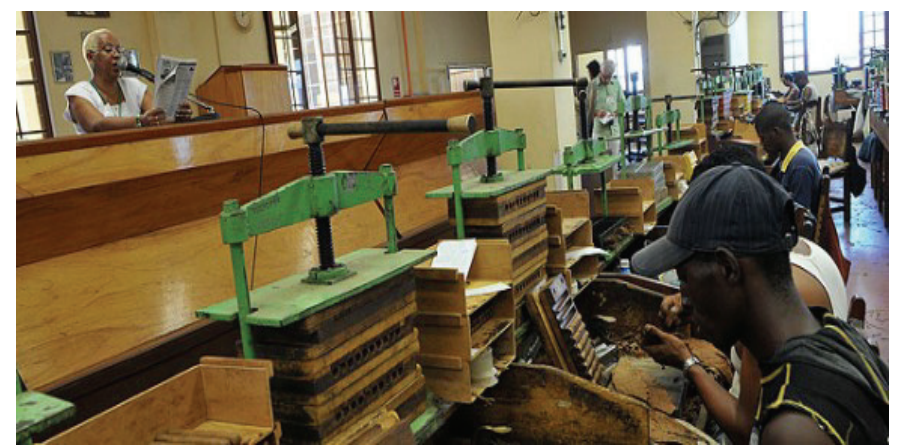

Foto: EFE http://www.cubadebate.cu/opinion/2012/11/25/lecturas-detabaqueria-patrimonio-cultural-de-la-nacion/\#.VhUsOJ7Lcy0

${ }^{2}$ Siehe Fernando Ortiz, Contrapunteo cubano del tabaco y el azúcar (1940; new edition La Habana: Ed. de Ciencias Sociales, 1983). 
Für diese bis heute gepflegten lecturas de tabaquería wählten sie Zeitungen und politische Schriften wie etwa Marx' Kapital aus, aber auch spannende Romane und die großen kanonischen Klassiker der Weltliteratur - darunter auch Shakespeares Tragödien und Komödien. Besonders beliebt unter den männlichen wie auch weiblichen tabaquieros war Romeo y Julieta. Das Produkt ihrer Arbeit nach Shakespeares Liebespaar zu benennen war daher nicht nur ein kluger Werbetrick, der sich des kulturellen Prestiges der Tragödie bediente, sondern eine literarische Huldigung an Shakespeare und seine Liebenden durch die, die sich mit deren Leidenschaft und deren Kampf gegen den väterlichen Despotismus identifizierten und überzeugt waren, dass die Exzellenz ihrer Zigarren der literarischen Exzellenz von Shakespeares Liebestragödie ebenbürtig sei.

Außerhalb Kubas sind diese lecturas de tabacquería und vor allem die Rolle die Romeo and Julia dabei spielten, nur wenig bekannt. Während einer Gastprofessur im mährischen Brno vor zwei Jahren machten mich jedoch Studenten auf einen ska oder reggae Song der populären Prager Band Zvírat (1991) aufmerksam, der diese Rezitationen von Romeo und Julia in der Zigarrenfabrik von Havanna feiert und lebendig werden lässt. Ihr Lead-Sänger impersoniert dabei die Rolle eines kubanischen Vorlesers und reflektiert über den Bann, in den Shakespeares Tragödie ihn und sein Publikum schlägt:

Hab jahrelang gelesen für die Zigarrendreher

Romeo und Julia In der Zigarrenfabrik

Jedes Monat las ich Romeo und Julia,

Ich las es ruhig, langsam, am Glas nippend

Las die Geschichte einer Liebe, die plötzlich endet

Und dann in die Zigarren eingewickelt wird

Den Zigarrendreher hab ich jahrelang

In der Zigarrenfabrik Romeo und Julia vorgelesen

In der Luft der Duft ihrer Liebe, denn wenn sie brennt

Brennt sie wir die Zigarre, schwindet die Liebe mit dem Rauch. [...]

Denn Schönheit schwindet so schnell wie Rauch [...]

Was die Liebe hier mit der Zigarre verbindet, ist die Intensität und die Kürze der Lust, ein Begehren, das sich nie ganz stillen aber sich in der Phantasie immer neu durchspielen und erneuern lässt. Das ist zugleich eine Allegorie der interkulturellen 
und intermedialen Zirkulation von Shakespeares Romeo und Julia: ein englisches Stück auf der Basis einer italienischen Novelle und in Italien spielend, vorgetragen in einer kubanischen Zigarrenfabrik und dies von einer tschechischen Band zum Reggae sound Jamaikas in Erinnerung gerufen... und das Ganze nun in Göttingen, Germany neu bedacht! Ein solch globaler Verkehr zwischen den Kulturen und Medien kann nur mit einem Stück funktionieren, das international so bekannt ist, dass selbst Menschen es kennen, die es noch nie gelesen oder gesehen haben; es kann nur mit einem Stück funktionieren, das bereits zum Mythos geworden ist einem Mythos, den jede neue Aufführung, in welcher Form auch immer, weiter bestätigt. Nicht alle Stücke Shakespeares haben einen solchen mythischen Status erlangt, aber Romeo und Julia, über die Jahrhunderte hinweg sein populärstes und beliebtestes Stück, in ungezählte Sprachen übersetzt, gezeichnet, gemalt, so oft auf die Bühne gebracht und verfilmt, zu symphonischer Musik gesetzt, in Ballette, Opern und Musicals verwandelt oder in Prosa nacherzählt und in andere Welten versetzt - denken Sie an Gottfried Kellers Romeo und Julia auf dem Dorfe! -, gehört sicher dazu. Und wenn es da ein Objekt in diesem Stück gibt, das es zusammenfasst, dann ist dies Julias Balkon. Es reicht, ,Balkon` zu sagen, und was einem dazu sogleich einfällt, ist der Balkon, auf dem Romeo und Julia ihr erstes Stelldichein haben (II.i) und auf dem sie sich nach ihrer ersten - und letzten - Liebesnacht trennen (III.v). Der Balkon ist das pars pro toto für das ganze Stück; er gewährleistet so sofortige Wiedererkennbarkeit und verkörpert für ein globales Publikum den Mythos der romantischen Liebe, wie er sich dem kulturellen Gedächtnis der Welt eingeschrieben hat.

\section{II.}

Wenn wir nun nach Havanna und Prag uns dem Text zuwenden, wie ihn Shakespeare 1597 in Druck gab, wartet eine Überraschung auf uns: von Julias gefeiertem Balkon, dem berühmtesten aller Balkone der Theatergeschichte, keine Spur! Keine Bühnenanweisung verweist auf ihn und weder Romeo noch Julia erwähnen in. Was wir vielmehr finden, ist eine "Gartenmauer", die Romeo überwinden muss (2.1.6 und 106) und Regieanweisungen, die nahelegen, dass Romeo vom Garten zu Julia aufblickt - vielleicht zu einem Fenster oder einer in die Wand eingelassene Loggia. In Begriffen elisabethanischen Bühnenbaus steht Romeo auf der platform stage und erscheint Julia auf der Oberbühne. Von einem Balkon im modernen Wortsinn, wie wir ihn von unserem Zigarrenkistchen und zahlreichen Inszenierungen und Verfilmungen kennen, ist da keine Rede - und 
kann auch gar keine Rede sein, denn das italienische Wort wurde erst einige Jahre nach Romeo und Julia im Englischen heimisch.

Es fehlt im Text, was die erste britische Romeo und Julia-Briefmarke, die zum Shakespeare-Jubiläumsjahr 1964, feiert -

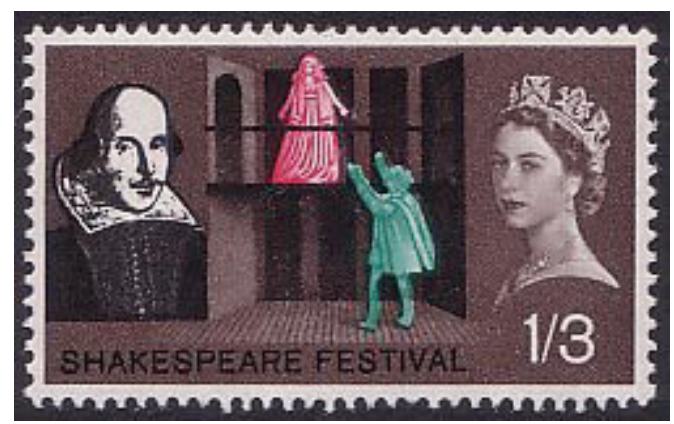

Thomson 1991: 5, 225-243 und Gurr: 1970: 96-98

oder was Julias Original-Geburtshaus in Verona auszeichnet und alljährlich viele Tausende von Shakespeare-Pilgern und romantischen Liebenden in die Città di Giulietta e Romeo pilgern lässt:

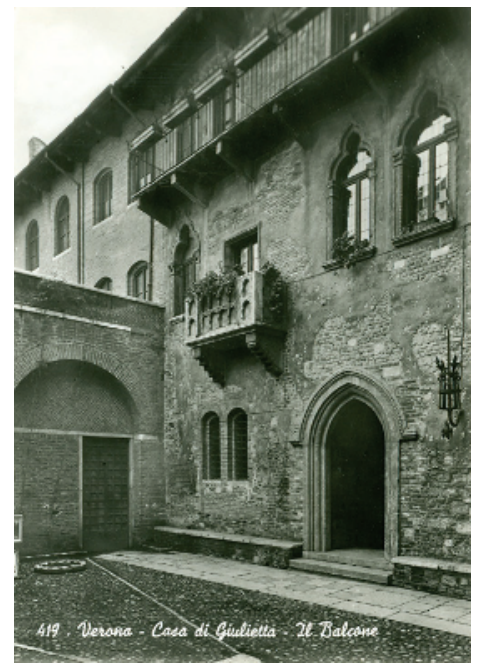

http://www.goethezeitportal.de/fileadmin/Images/wd/projekte-

pool/italien/reisestationen/verona/verona_romeo_julia/Casa_di_Giulietta_419_500x717_.jpg

Diese Casa di Giuglietta ist natürlich eine Fiktion und alles andere als authentisch. Wäre sie das, müsste sie ja in Siena liegen, wo, mehr als zwei Jahrhunderte vor Shakespeare, der Novellendichter Masuccio aus Salerno seine Geschichte angesie- 
delt hatte. Und doch folgen Touristen seit dem 18. Jahrhundert in wachsenden Zahlen dem Romeo und Julia-Trail quer durch Verona, von Julias Haus in der Via Cappello zu dem Romeos und zu ihrer Gruft in San Francesco al Corso. Da gab es allerdings ein kleines Problem: Dem angeblichen Familiensitz der Capuleti - eine „schmutzige Kneipe“, wie Heine befand, oder „a most miserable inn“, wie Dickens ihm beipflichtete - fehlte der Balkon. Bis schließlich die weisen Stadtväter Veronas in den späten 1930er Jahren beschlossen, ihre Gäste nicht weiter zu enttäuschen und zur optimalen touristischen Vermarktung des Shakespeare'schen Liebespaares dem Haus einfach einen Balkon zu verpassen. Und sie ließen sich dabei von Hollywood inspirieren, nämlich dem schönen Pseudo-Renaissance-Balkon in George Cukors erstem Romeo und Julia-Tonfilm von 1936. 1972 stifteten sie dazu dann noch eine hübsche Statue der Julia, um die Aura des Authentischen weiter zu vertiefen. Ihr nackter Busen ist inzwischen hoch poliert von den innig rituellen Berührungen romantischer Liebender aus aller Welt - darunter, so will es ein Gerücht, Nicolas Sarkozy und Carla Bruni auf ihrer Hochzeitsreise. Sie alle wallen zu Julias Schrein und geben sich ausufernden Ritualen hin - vom Briefeschreiben an "Julia, Verona, Italy“, auf die dann ein gut organisierter "Club di Giulietta“ antwortet, über das Bekleben des Gebäudes mit Kaugummi oder der Befestigung von ewige Liebe symbolisierenden Schlösser bis hin zum Austausch der Hochzeitsschwüre und -küsse auf dem Balkon selbst zum derzeitigen Preis von 800 Euro.

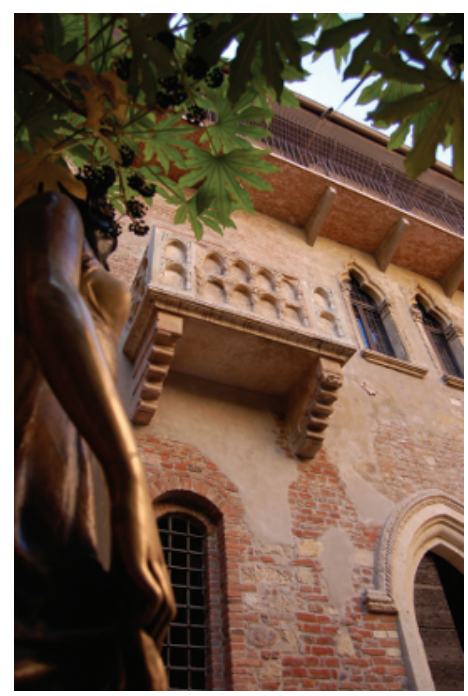

Balcone della casa di Giulietta

https://commons.wikimedia.org/wiki/File:Balcone_della_casa_di_Giulietta.jpg 


\section{III.}

Unabhängig davon, wie wir uns Julias Balkon vorstellen, wird er doch immer seinen ,liminalen' Charakter bewahren - ein Raum zwischen oben und unten und zwischen drinnen und draußen. Solche liminale oder Zwischenräume spielen eine wichtige Rolle in vielen Shakespeare-Dramen: Denken Sie bloß an die Erscheinung des Geistes auf der Brüstung von Hamlets Schloss! Hier geht es immer um mehr als räumliche Beziehungen, denn diese sind immer mit kulturellen symbolischen Codes aufgeladen: die vertikale Achse als Gegensatz zwischen dieser Welt und dem Jenseits, zwischen niedrigeren menschlichen Instinkten und dem Streben nach höheren, erhabenen Werten, zwischen sexuellem Begehren und reiner, platonischer oder romantischer Liebe; die horizontale Achse als Gegensatz zwischen öffentlichen, privaten und intimen Räumen. In diesem Sinn stellt Julias Balkon in der Tat das Zentrum des Stücks dar. Er steht zwischen den öffentlichen Räumen sozialer und politischer Konflikte - die Straßen und Plätze Veronas, der Ballsaal - und der Privatheit des bedrückenden Familienszenarios und der radikalen Intimität von Julias Schlafgemach und der Gruft der Liebenden in der Krypta. Wir bewegen uns sozusagen vom Balkon zum Bett und weiter zur Gruft, wo der Liebestod die Liebenden schließlich vereint und trennt. Die Zentralität des Balkons wird weiter dadurch unterstrichen, dass er den Schauplatz von drei Szenen abgibt: die Balkonszene der Liebenden in II.i, Julias leidenschaftlich ungeduldiges Hochzeitslied in III.ii und die Abschiedsszene im Morgengrauen nach der Liebesnacht, III,v. Die zwei Balkondialoge dramatisieren die Liminalität ihres Schauplatzes dadurch, dass sie ihn in entgegengesetzten Richtungen spiegeln: der erste aus eine Perspektive vom Garten zum Balkon und zur Schlafkammer; die zweiter von diesen zum Balkon und in den Garten. Es ist vor allem in diesen beiden Szenen mit ihrem raum-symbolischen Gewicht, dass Shakespeares Liebende zu ihrer eigenen Sprache finden, um die Liebe, die ihnen widerfährt, auszudrücken - eine absolute und grenzenlose Liebe, eine Verschmelzung ihrer Körper, Gefühle und Gedanken, ein Ineinander von eros und thanatos ebenso wie ihrer Ich-Identität, für die sie kühne Sprachbilder finden, die alle Konventionen eines herkömmlichen poetischen discours amoureux sprengen. 


\section{IV.}

Kein Wunder, dass die zahlreichen Künstler, die Szenen aus Romeo und Julia gezeichnet oder gemalt haben, immer wieder auf den Balkon als zentrales Symbol fokussierten. ${ }^{3}$ Die Kunstgeschichte zu diesem Stück setzte im späten 18. Jahrhundert ein, als der Balkon schon längst - nämlich seit den Inszenierungen von Thomas Otways Romeo und Julia-Bearbeitung von 1680 - seinen festen Platz auf der Bühne gefunden hatte, und sie gipfelte im 19. Jahrhundert, als der Kult der romantischen Liebe, der grande passion und des Liebestods die erotischen Phantasien des Bürgertums beherrschte. Man denke nur an Wagners Tristan und Isolde (1865) oder an Romane wie Flauberts Madame Bovary (1856-57) oder Tolstoy's Anna Karenina (1873-78)! Was die Maler daran reizte, war auch der Glanz des italienischen Schauplatzes mit seinem Palast und der Stadt und Landschaft des Veneto im Hintergrund. Schließlich gehören Balkone und Loggias zum festen Bestand eines sinnlich-warmen Südens und nicht des grimmigen Nordens von Hamlets Dänemark oder Macbeths Schottland.

Schauen wir uns ein paar dieser Bilder an und beginnen wir mit Francesco Hayez' „L'ultimo bacio di Romeo e Giulietta“ in der Szene 3.5 (1823):

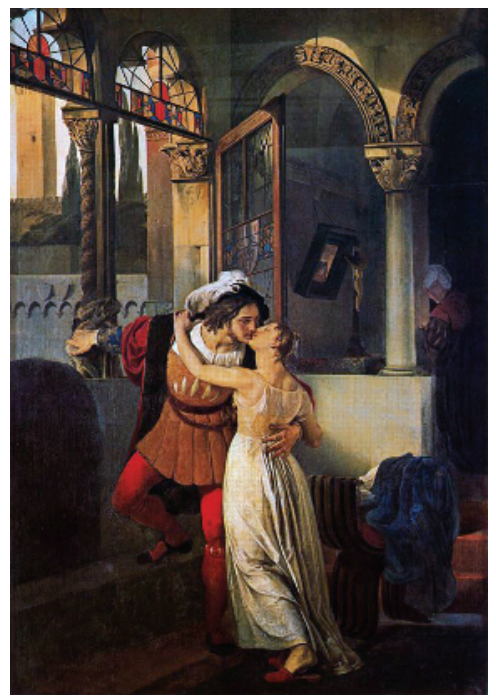

Francesco Hayez, L'ultimo bacio dato a Giulietta da Romeo https://commons.wikimedia.org/wiki/File:Francesco_Hayez_053.jpg

\footnotetext{
3 See James Fowler, „Picturing Romeo and Juliet“, in Stanley Wells (ed.), Shakespeare Survey, 49
} (1996), pp. 111-130. 
Romeo in voller Kleidung ist schon auf dem Weg zum Balkonfenster, während Julia, noch im Schlafrock, ihn vom Geländer abzuhalten versucht. Offensichtlich ist dieser venezianische Maler mehr am Renaissance-Glamour des Balkonbogens und den feinen Farbnuancen des morgendlichen Himmels auf den Zypressen und dem phallischen Turm vor dem Schlafgemach interessiert denn an einer Analyse der Gefühle der Liebenden.

Unser nächstes Bild kennen wir schon vom kubanischen Zigarrenkistchen. Der österreichische Maler Hans Makart, zu seinen Zeiten international hochberühmt, schuf diese Balkonszene 1872-76 als Teil eines Zyklus von Gemälden zu Romeo und Julia, und sie gefiel Kaiser Franz Joseph so gut, dass er sie sogleich für seine Sammlung aufkaufte:

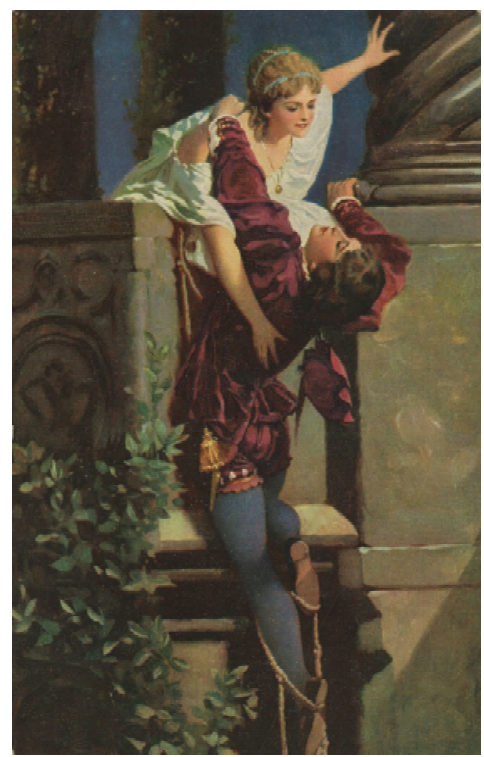

Hans Makart, Romeo und Julia www.zeno.org/nid/20000729167

Ihr Format dramatisiert die vertikale Achse des Abstiegs, und zu diesem Drama trägt auch die schier akrobatische Koordination der Glieder, der Brüstung und der Strickleiter dieses spontan frischen und entschieden jungen Liebespaars bei, deren junge Liebe so vital und saftstrotzend erscheint wie die das Blattwerk der Kletterpflanze, die nach oben strebt. Wir werden noch lange warten müssen, bis wir dann in Zeffirellis Film der siebziger Jahre einem vergleichbar jungen und ungestümen Liebespaar wiederbegegnen werden. 
Die Perspektive hier, wie in meinem nächsten Bild, ist von der Höhe außen, und in beiden Fällen betont dieser schwindelnde Blickwinkel die dramatische Körpersprache sich verklammernder und baumelnder Körperteile. Im Fall von Ford Madox Browns prä-raffaelitischem Gemälde von 1871 ist jedoch die Körperhaltung der Liebenden unbeholfen und ungelenk eher als elegant wie bei Makart:

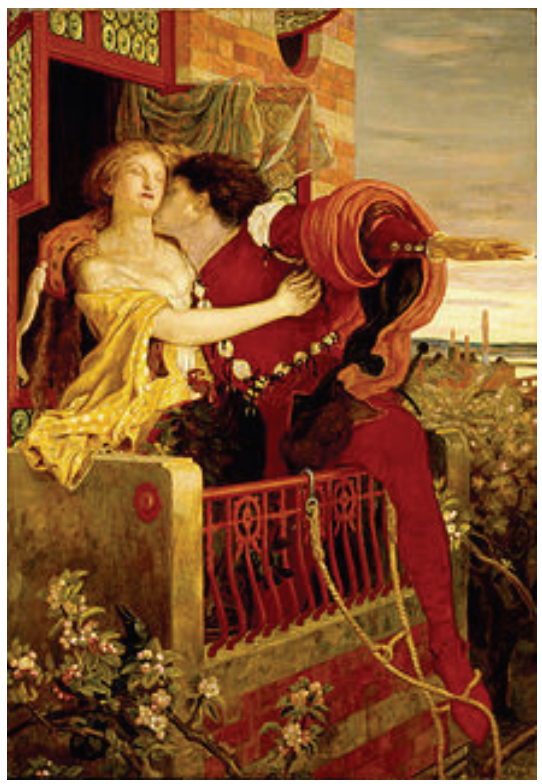

Ford Madox Brown, Romeo and Juliet https://commons.wikimedia.org/wiki/File:Romeo_and_juliet_brown.jpg

Mehr noch als Makart dramatisiert Ford Madox Brown die vertikale Achse: Der Balkon zusammen mit Romeos freiem Bein, der baumelnden Strickleiter und den aus der Tiefe heraufwachsenden Blütenzweigen schweben bedenklich über dem Abgrund der der Stadtlandschaft Veronas. Dies steigert die Dramatik der Situation, den Heroismus der Liebenden und die tragische Fallhöhe, die sie bedroht.

Frank Dicksees „Romeo and Juliet“ (1884), im altmeisterlichen Stil der Mitglieder der Royal Academy gemalt, ist wohl das romantischste aller viktorianischen Bilder von Shakespeares mythischem Liebespaar. Kein Wunder, dass es vor drei Jahren, am Valentinstag 1012, bei einer öffentlichen Umfrage mit $71 \%$ der Stimmen zum romantischsten aller Bilder in britischen Museen und Galerien 
gewählt wurde ${ }^{4}$ und so manchen Buchumschlag von Ausgaben des Stücks schmückt:

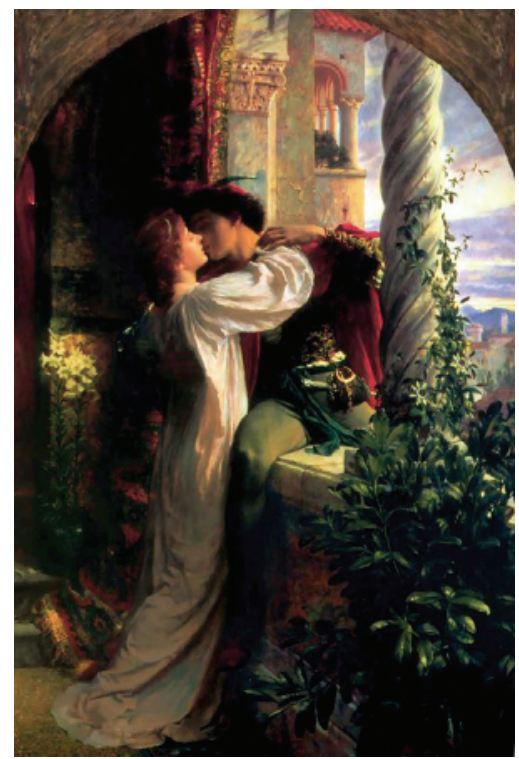

Frank Dicksee, Romeo and Juliet

https://commons.wikimedia.org/wiki/File:DickseeRomeoandJuliet.jpg

Es zeigt den rührendsten Augenblick der letzten Umarmung, des zärtlichen eher als leidenschaftlichen Abschiedskuss und verleiht ihm durch die Rahmung mit Girlanden blühenden Grüns und dem großen Rundbogen die Aura einer Heiligenvita. Nichts stört hier die Versenkungen der Liebenden ineinander; hier drängt keine Amme zur Eile, wie bei Shakespeare, der Abstieg vom Balkon scheint keine Gefahren zu bergen und das Licht der Morgendämmerung über der malerischen italienischen Landschaft dräut nicht, sondern ist nur schön.

Mit Dicksees Gemälde haben wir den Gipfel des viktorianischen Kults einer absoluten Liebe jenseits aller banalen oder feindlichen Realität erreicht. Das war nicht mehr zu überbieten, und so kehrten zukünftige Maler Julias Balkon romantischer Liebe eher den Rücken oder machten sich darin, ihn zu demontieren. Wo sie auf ihn überhaupt noch verwiesen, dann war dies in der Regel im Geist der Parodie oder des Pastiche - wie zum Beispiel in Lance Thackerays eleganter Farblithographie von 1908, „Romeo and Juliet-Balcony Scene at Shepheard's Hotel, Cairo", die die Liebenden auf ihrem Balkon in Verony in ein touristisches

\footnotetext{
${ }^{4}$ Vgl. http://www.dailymail.co.uk/news/article-2099615/Is-romantic-painting-Oil-depiction-RomeoJuliet-voted-best-Britain.html
} 
Kairo versetzt und ihren Moment transzendentaler Liebe in eine moderne Geschichte von Flirt und Entfremdung übersetzt:

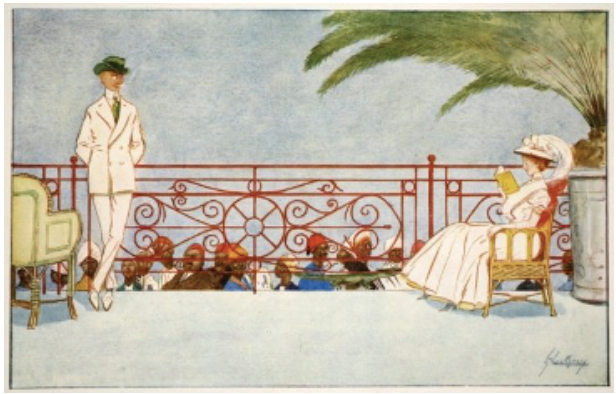

Lance Thackeray, „Romeo and Juliet-Balcony Scene at Shepheard's Hotel, Cairo“.

The Light Side of Egypt. London: Adam and Charles Black,1908.

Oder, ein letztes Beispiel, Annie Leibovitz Photograpie „Auf den Schwingen Cupidos" für das Vogue Modemagazin, das im Dezember 2008 unter dem Titel "The Love of a Lifetime“ eine zwölfseitige Photostrecke von Romeo und JuliaBildern in prächtiger Kostümierung vor glanzvollen italienischen RenaissanceSettings ihren modebewussten Lesern anbot: ${ }^{5}$

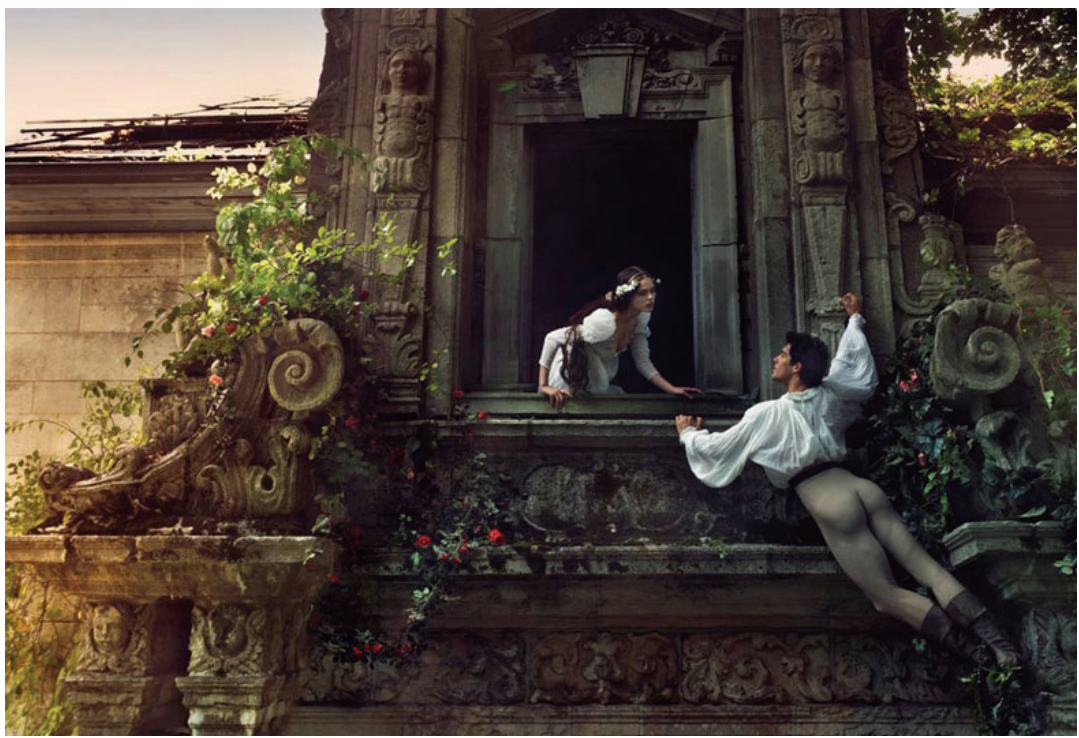

Annie Leibovitz, „On Cupid's Wings“. Vogue (December 2008)

\footnotetext{
${ }^{5}$ See http://www.mymodernmet.com/profiles/blogs/romeo-and-juliet-by-annie
} 
Das ist ganz bewusst Kitsch - oder wie Annie Leibovitz' Geliebte Susan Sontag gesagt hätte: Camp. Die Photoinszenierung zitiert nicht nur alle Konventionen der romantischen Balkonszene - auch wenn der Balkon hier eher eine Fensternische ist - sondern inszeniert die Schönheit der Liebenden mit überschwänglichem Gusto. Diese überzogene Theatralität lässt das Bild wie ein Standphoto aus einem der romantischeren Filmversionen des Stücks, der Zeffirellis etwa, erscheinen, und die Besetzung der Rollen mit Coco Rocha als Julia und Roberto Bolle als Romeo, beide berühmte Photomodelle und der letztere auch berühmt als Balletttänzer, feiert die Schönheit der Körper, Kostüme und Bewegungen der Liebenden. Hier, ebenso wie in einem Werbeclip der Modekette H\&M, die sich der Balkonszene bedient, ihre hippsten neuen Jeans raffiniert sexy ins Bild zu setzen, kippen Shakespeares Liebestragödie und der Mythos romantischer Liebe vollends in die Pop Art kommerzieller Warenästhetik um - in eine Oberfläche, die keine Tiefe mehr kennt, in ein Simulacrum, an dessen Original niemand mehr zu glauben vermag. ${ }^{6}$

\section{V.}

Die Filmgeschichte von Juliets Balkon ist fast so lang wie die Geschichte des Kinos selbst. Kaum war das neue Medium im späten neunzehnten Jahrhundert erfunden, da wandte es sich schon in einer Reihe von Stummfilmen Julias Balkon zu. Das kulturelle Prestige Shakespeares zusammen mit der Popularität des Stücks empfahlen es dem neuen Medium, mit ihm dem Ruch billigen Jahrmarktspektakels hinter sich zu lassen und sich als neue Kunstform zu etablieren. An die zehn Stummfilmversionen wurden in Frankreich, Italien, England und den USA gedreht, und es ist bis heute unter allen Stücken Shakespeares der absolute Liebling der Leinwand geblieben. Da Stummfilme ohne Shakespeares Poesie auskommen müssen, haben sie immer wieder versucht, diesen Verlust durch den besondere visuellen Reiz von Originalschauplätzen zu kompensieren, wie etwa in Gerolamo Lo Savio 1911 in Verona gedrehten Film; und da sie sich auf einzelne Schlüsselszenen konzentrieren mussten, wurde die Balkonszene schnell zur obvious choice der Stummfilm-Regisseure.

Diese visuellen Konventionen setzten sich in die Ära schwarz-weißer Tonfilme fort, die für Romeo und Julia mit George Cukors Film von 1936 begann. Er wurde zwar in Hollywood gedreht, doch schickte Metro Goldwyn Mayer ein Expertenteam nach Verona, um das historische Ambiente richtig hinzukriegen und mit

\footnotetext{
${ }^{6}$ See http://www.youtube.com/watch?v=ZKdWFC9T9ps
} 
Bildmaterial aus der italienischen Renaissance die Kostüm- und Bühnenbildner zu inspirieren. Das Ergebnis dieser Bemühungen und historische Authentizität war allerdings kaum überzeugend - und sei das auch nur, weil der siebenundvierzigjährige Leslie Howard als Romeo und die sechsunddreißigjährige Norman Shearer als Juliet nicht wirklich überzeugen konnten. Ein Standphoto mag reichen, den Mangel an jugendlicher Leidenschaft in ihren steifen Gesten zu belegen.

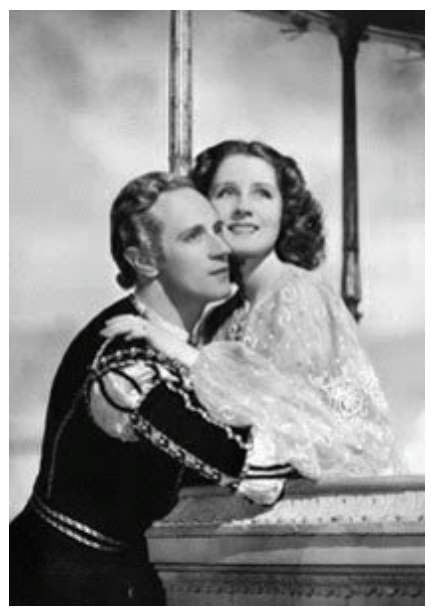

Romeo and Juliet 1936 Foto von George Hurrell

Dem Farbfilm nach dem Zweiten Weltkrieg gelang es viel besser, das visuelle und dramatische Potential von Shakespeares Hohem Lieder der romantischen Liebe zur Geltung zu bringen. Betrachten wir kurz Ausschnitte der ersten Balkonszene in drei dieser Farbfilme, denen Renato Castellanis, Franco Zeffirellis and Baz Luhrmanns!

Renato Castellanis italienisch-englische Koproduktion von 1954 geht noch einen entscheidenden Schritt weiter in der Italienisierung seines Romeo und Julia Films: Er dreht ihn ausschließlich in Italien und versucht in seinen prachtvollen Bildsequenzen eine Quintessenz von italianità, von italienischem quattrocento zu destillieren. Dazu reicht ihm Verona allein ganz offensichtlich nicht, und so bewegen wir uns in seinem Film wie auf einer Bildungsreise von Verona nach Venedig, Siena, San Quirico d'Orcia und anderen pittoresken Schauplätzen vor allem der Toskana. Julias Balkon belegt dies eindrücklich: es ist nicht der der Casa die Giulietta in der Via Cappello in Verona, sondern die viel imposantere Struktur im gotischen Hof der $\mathrm{Ca}^{\prime}$ d'Oro, einem der schönsten Paläste am Canal Grande Venedigs. Hier ist er: Dieser Balkon ist kein idyllisch romantischer Ort. Er ist von gewaltigen Dimensionen in seinen tiefen Gewölbebögen, die auf einen dunklen, 
geschlossenen Hof blicken, und die weite Treppe mit mächtigem Marmorgeländer, die zu ihm hinaufführt, verlangt dem jungen Liebenden zwar keine besondere sportliche Leistung ab, beeindruckt ihn aber schon durch seine gigantischen Ausmaße. Zudem ist dieser Balkon wie ein Verließ von schweren Eisengittern umschlossen - ein visuelles Leitmotiv der sozialen Unterdrückung, das den ganzen Film durchzieht. ${ }^{7}$ So finden die Liebenden hier keinen Weg, sich durch diese marmornen und eisernen Barrieren zu berühren, zu umarmen oder zu küssen; sie bleiben voneinander getrennt und können nur durch ihre poetischen Worte die Distanz überbrücken. Ihre Liebe bleibt eine romantische, auf ihre romantische Liebe ist eine, die das eiserne Gesetz gesellschaftlichen Zwangs, der Macht des Patriarchats, des Feudalismus, der Kirche und der Sippenfehde unbarmherzig unterdrückt.

Der Film brachte Castellani 1954 den Leone d'oro in Venedig ein, floppte aber an den Kinokassen; offensichtlich war dem Publikum der Balkon dann doch nicht romantisch genug. Zeffirellis anglo-italienische Romeo und Julia Produktion von 1968 erwies sich dagegen sowohl bei den Kritikern wie an den Kassen als überwältigender Erfolg und gilt bis heute als die klassische Verfilmung von Shakespeares Liebestragödie. Der Unterschied kann nicht im visuellen Reiz der italienischen Schauplätze liegen, denn beide plündern gekonnt die architektonischen und künstlerischen Schätze der italienischen Renaissance - Zeffirelli für seinen Balkon den des Palazzo Borghese in Artena, südlich von Rom. Der entscheidende Unterschied liegt vielmehr in dem, was Zeffirellis Untertitel herausstellt, „Romeo und Julia: die Teenage-Liebenden von Verona“, und damit eine Filmversion ankündigt, die sich in Einklang mit der 68er Jugendkultur in ihrer Revolte gegen die autoritäre, Vietnam Krieg führende ältere Generation findet. Endlich einmal ein Liebespaar so jung, wie es sich Shakespeare vorstellte: Olivia Hussey ist sechzehn und Leonard Whiting wenig älter. Das gibt schon der Balkonszene - nach Zeffirelli der „Dreh- und Angelpunkt“ des Stücks ${ }^{8}$ - einen ganz neuen Glanz. Der weitgespannte, hohe Balkon, von allen Seiten von Grün überwuchert, erlaubt es den Liebenden sich zu berühren und ihre jugendliche Energie sowohl physisch als auch verbal auszuagieren, während sie sich auf dem Balkon hin und her bewegen und sich mit jedem Wortwechsel immer neu vereinen und trennen. ${ }^{9}$ Auf diesen Balkon führt keine Treppe, aber der ungestüme

\footnotetext{
${ }^{7}$ See Patricia Tatspaugh, „The Tragedies of Love on Film“, in: Russell Jackson (ed.), The Cambridge Companion to Shakespeare on Film (Cambridge: CUP, 2000), pp. 135-159, here p. 140.

${ }^{8}$ Zeffirelli with reference to his theatre production of Romeo and Juliet at the London Old Vic, 1960, quoted in Cavecchi.

${ }_{9}$ Peter S. Donaldson, Shakespeare Films / Shakespearean Directors (Boston: Unwin Hyman, 1990), p. $176 f$.
} 
Liebhaber braucht auch keine: er schwingt sich mit akrobatischer Bravour auf dem Baum hoch, um seinen ersten leidenschaftlichen Kuss zu erlangen. Das Zusammenspiel ihrer Augen und Lippen bebt von erotischer Spannung, und Nino Rotas sehnsuchtsvoll romantische Musik unterstreicht dies noch. Sie setzt hier das musikalische Leitmotiv ihrer Liebe, den Song "What is youth" fort, der in der Ballszene als Begleitmusik zu ihrer Liebe auf den ersten Blick erklang. Musik ist schließlich die wahre Sprache romantischer Liebe, wie sie schon die Komponisten des 19. Jahrhunderts in ihren Opern und Symphonien feierten und wie Franco Zeffirelli als erfolgreicher Opernregisseur nur zu gut wusste.

Mein letzter Film-Balkon sprengt alle Erwartungen. Wie Castellani und der Zeffirelli bedient sich auch Baz Luhrmanns Holywood Produktion dazu eines authentischen historischen Gebäudes, aber dieser Balkon gehört nun nicht zu einem italienischen Renaissancepalast, sondern zum Castillo de Chapultepec, das seit dem neunehnten Jahrhundert auf seinem Hügel über Mexico City steht. Als solcher ist er Teil einer geographischen und kulturellen Transposition des Geschehens vom italienischen Verona zu einem lateinamerikanischen „Verona Beach", das ebenso an Mexiko wie an Kalifornien oder ein kubanisiertes Miami denken lässt. Darüber hinaus ist da nicht nur der historische Balkon, sondern, ihm zugefügt und den Raum in der Vertikale erweiternd, ein moderner Swimming Pool mit allem was dazu gehört, von Pool Möbeln bis zu bewegungssensitiven Sicherheitsmeldern und security guards. So reduziert sich für Leonardo DiCaprios Romeo und Claire Danes Julia die petrarkistische Höhendifferenz zwischen angebeteter Dame und werbenden Verehrer von Anfang an: er fällt vom Spalier, sie stürzt zu ihm herab, und gemeinsam tauchen sie ins tiefe Wasser des Pools, um die Intimität ihrer Liebe vor der sozialen Kontrolle zu verbergen. Postmodern wie dieses ständigen Überkreuzungen kultureller Markierungen zwischen der Renaissance und der Gegenwart, Italien und Lateinamerika, klassischer Tragödie und Hollywood Melodrama, Action Film, college romance oder Video Clip erscheinen, so sind sie doch keineswegs willkürlich oder nur parodistisch. Der scheinbar völlig deplatzierte Swimmingpool, zum Beispiel, ist Teil eines visuellen Leitmotivs, das in der Ballszene eingeführt wird, in der sich Romeos und Julias Liebe im ersten gegenseitigen Erblicken durch ein Aquarium entzündet; er erscheint als Miniaturversion von Julias poetischen Bildern für ihre Liebe "boundless as the sea“ (2.1.176), und er verbindet sich noch mit der religiösen Symbolik von Taufe und Reinigung, auf die auch Julias Engelsgewand hier anspielt. Denn so sehr auch Luhrmanns postmoderne Verfremdungen Shakespeares Poesie unterminieren, sexualisiert sein Film nicht nur die romantische Liebe weit über alle romantische Konventionen hinaus, er sakralisiert sie auch - und das für ein Publikum, für das 
römisch-katholische Bilder und Rituale ansonsten nur noch bizarr erscheinen. Kein Wunder also, dass Nellee Hoopers Soundtrack nicht nur Rock und Reggae zu bieten hat: Die Balkonszene ist mit Des'rees Soul Song „Kissing You“ unterlegt, der in einer sehnsüchtigen Instrumentalversion zum klimaktischen Kuss hinführt, und über den letzten Bildern des Films schwellen Kirchenmusik und der „Liebestod" von Wagners Tristan und Isolde auf.

\section{VI.}

Im Rückblick auf mehr als hundert Jahren Romeo und Julia auf der Leinwand könnte man meinen, dass Shakespeares Liebestragödie erst als Movie-Melodrama so recht zu sich kam. Es gibt aber eine andere Form von Melodrama - und diese

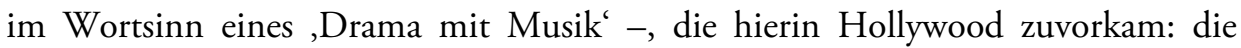
zahlreichen Opern und Bühnenmusiken zu dieser ,Mutter aller Romanzen', von Berlioz‘ ,Dramatischer Symphonie‘ und Tschaikowskys ,Ouvertüre' zu Gounods Romeo e Juliette Oper und Prokofjews Ballett und weiter zu Leonard Bernsteins Bühnen- und Film-Musical, der West Side Story. Diese Welle von Vertonungen schwoll erst an, als im neunzehnten Jahrhundert der Mythos der grande passion alle Medien zu überspülen begannt, vor allem aber die Musik und den Tanz. Um es mit Walter Pater zu sagen: Jede romantische Liebe strebt nach Musik - der Tiefe und Intensität ihrer Gefühle jenseits aller Worte.

Wir haben hier natürlich nicht mehr die Zeit, all diese musikalischen Balkone zu erklimmen, von denen einige besonders hoch und erhaben sind. Erlauben Sie mir vielmehr aber doch noch, Ihnen dazu zwei neuere Ballette vorzustellen, die auf Kompositionen des 19. Jahrhunderts zurückgreifen.

Mein erstes Beispiel führt uns zurück nach Havanna, wo 2003 Alicia Alonso mit ihrem international gefeierten Ballet Nacional de Cuba ein zwanzig-minütiges Romeo y Julieta Kondensat, Shakespeare y sus masquarás, unter freiem Himmel auf der Plaza de las Armas im Herzen der Altstadt aufführte. Das was eine Art MetaDrama, in dem Shakespeare selbst auftritt und seine Figuren begleitet, wie hier in der Balkonszene, die eine der prächtigsten Loggien der Stadt ins Spiel einbezieht. Alicia Alonso legt ihrer Choreographie eine Orchesterversion von Charles Gounods Roméo et Juliette-Oper zugrunde, der französischen Antwort auf Wagners LiebestodOper Tristan und Isolde von 1865, zwei Jahre vor Gounod. Hier lenkt nichts vom Zusammenspiel der jungen Körper ab. Sonst ist wenig zu sehen außer dem Unten und Oben von ebenem Boden und Loggia. Da gibt es auch keine Worte, und man vermisst sie nicht, denn Gounods Musik ohne ihr recht banales französisches Libretto ist bewegender als mit ihm. Und hinter dem Balkon ist nichts, keine 
geschwätzige Amme und keine widerständigen Eltern; das junge Paar, ineinander versunken, scheinen von nichts zu wissen als ihrer Liebe. Sie tanzen zunächst auf ihren verschiedenen Ebenen; dann steigt sie aus eigenem Antrieb zu ihm hinab, statt zu warten, bis er zu ihr hinaufklettert, und der folgende pas de deux, der in ihrem Kuss gipfelt, destilliert die Quintessenz junger romantischer Liebe als einer transzendentalen Erfahrung jenseits aller gesellschaftlichen und politischen Kontexte - und das ausgerechnet in Kuba, das reichlich Anlass für Konfliktszenarien geben würde.

Mein zweites Ballett-Beispiel geht auch von einer Vertonung von Shakespeares Liebestragödie aus dem neunzehnten Jahrhundert aus - von Hector Berlioz' symphonie dramatique Roméo et Juliette von 1839, nicht zufällig ein wichtiges Modell für Wagners Tristan und Isolde. Berlioz hatte sich auf den ersten Blick in Romeo und Juliet verliebt, als er 1827 dem Stück im englischen Original begegnete. Es war ihm sofort klar geworden, dass eine regelrechte Oper nicht hinreichen würde, „das Drama dieser Leidenschaft so schnell wie der Gedanke, so glühend wie Lava und so strahlend rein wie ein Engelsblick" zu fassen. ${ }^{10}$ Er musste also eine neue Form erfinden, die die Oper mit der Symphonie verbindet, eine „dramatische Symphonie“, die die Höhepunkte des Stücks emotional auslotet. Eine davon ist die Balkonszene, Berlioz' eigener Favorit, ein schier endloses Adagio. ${ }^{11}$

Als die Berliner Choreographin Sasha Waltz 2007 das Angebot erhielt, Berlioz' dramatische Symphonie für die Pariser Opéra de la Bastille als Ballett zu inszenieren, entschied sie sich, die ursprünglich Struktur zwischen Oper und Symphonie zu bewahren und in ihr emotional expressives Körpertheater zu übersetzen. Dazu ließ sie sich ein hoch-abstraktes, kubistisches Bühnenset in Schwarz-Weiß bauen, das aus kaum mehr besteht als zwei riesigen Plattformen, eine über der anderen und die obere nach Bedarf winklig anzuheben. Wenn wir in diesem Ballett die Balkonszene erreichen, überrascht uns gleich eine Abwesenheit: Hier gibt es keinen Balkon! Romeo und Julia treffen sich auf einer Ebene, sozusagen auf Augenhöhe, und damit bricht von vornherein die ganze platonische oder petrarkistische Raumsymbolik von Oben und Unten zusammen. Die Beiden sind gleich in ihrer Liebe, und wenn der eine oder die andere gleicher sein sollte als der oder die andere, dann ist dies Julia eher als Romeo. Immer wieder gehen die Bewegungsimpulse von ihr aus, und er nimmt sie dann auf in diesem Spiel der Selbsterfindung in neuen Bewegungsformen, die wenig von den konventionellen des klassischen pas de deux an sich haben. Sie erfinden zusammen und für sich eine neue Körperspra-

${ }^{10}$ The Memoirs of Hector Berlioz, ed. and translated by David Cairns (New York: Alfred A. Knopf, 2002), p. $72 \mathrm{f}$.

${ }^{11}$ The Memoirs, p. 526. 
che, so wie ja auch bei Shakespeare Julia eine neue Sprache der Liebe erfindet und sie Romeo vermittelt. Sie erfinden sogar neue Arten und Weisen des ,Füßelns' und Küssens, ihre ebenso leidenschaftliche wie verspielte Liebe auszudrücken. Nur gegen Ende verdunkelt sich diese Feier junger Liebe im Einklang mit Berlioz romantischer Musik, und dann beginnt sich langsam die obere Plattform mit Julia auf ihr zu heben - nicht aber, um einen Balkon zu formen, sondern eine Trennwand, die die beiden Liebenden voneinander trennt wie die von Pyramus und Thisbe bei Ovid (und in Shakespeares Sommernachtstraum).

Mit diesem Durchstreichen von Julias Balkonen endet meine Geschichte von dessen Aufstieg und Niedergang. Was diese Geschichte zeigt, ist, dass sie der Mythos der romantischen Liebe, den sie symbolisiert, erstaunlicherweise recht unbeschadet überdauert hat. Dies ließe sich auch für die Geschichte des Balkons im Sprechtheater zeigen, „had I but world enough and time“ sie noch einzuflechten. Auch hier könnten wir beobachten, wie Julias romantischer Balkon sehr viel nüchternen und prosaischeren Bühnenaufbauten weicht oder in minimalistischer Abstraktion verschwindet. Peter Brook z.B. ließ es schon 1947 mit einer weißen Wand als Balkon bewenden, und Jette Steckel in ihrer noch laufenden Inszenierung am Hamburger Thalia Theater kommt mit einem kleinen Treppenabsatz aus. In anderen Fällen, in denen die Inszenierung das Stück in ein aktuelles politisches Szenario übersetzt, verwandelt sich der Balkon z.B., wie in der West Side Story, in eine offene Feuertreppe Manhattans. Aber selbst dies entmythologisiert die romantische Liebe nicht wirklich, sondern lässt sie nur umso heller erstrahlen. 


\section{Bibliographie}

Berlioz, Hector. The Memoirs of Hector Berlioz. Hg. u. Übers. David Cairns. New York: Alfred A. Knopf, 2002.

Donaldson, Peter S.. Shakespeare Films/Shakespearean Directors. Boston: Unwin Hyman, 1990.

Fowler, James, „Picturing Romeo and Juliet“, Shakespeare Survey, 49, Hg. Stanley Wells, 1996.

Gurr, Andrew. The Shakespearean Stage. 1574-1642. Cambridge: Cambridge University Press, 1970.

Ortiz, Fernando. Contrapunteo cubano del tabaco y el azúcar. New edition La Habana: Hg. de Ciencias Sociales, 1983.

Tatspaugh, Patricia, „The Tragedies of Love on Film“, The Cambridge Companion to Shakespeare on Film, Hg. Russell Jackson, Cambridge: CUP, 2000. 135-159.

Thomson, Leslie. „Window Scenes in Renaissance Plays: A Survey and Some Conclusions“, Medieval \& Renaissance Drama in England. Vol.5 (1991): 225-243.

\section{Elektronische Quellen}

http:/www.dailymail.co.uk/news/article-2099615/Is-romantic-painting-Oildepiction-Romeo-Juliet-voted-best-Britain.html

http://www.mymodernmet.com/profiles/blogs/romeo-and-juliet-by-annie http://www.youtube.com/watch?v=ZKdWFC9T9ps 